obstruction and low rates of comorbidities) had a lower mortality, but no significant survival difference was found between phenotypes 1, 3 and 4 . Because subjects belonging to various phenotypes had marked differences in age at inclusion (median age 58-73 yrs) and because the observation period was relatively short, we hypothesised that age of death would be different among the phenotypes. Indeed, median (interquartile range) age of death was: 62 (58-68) yrs for phenotype 1; 77 (66-83) yrs for phenotype 2; 67 (58-69) yrs for phenotype 3; and 76 (74-79) yrs for phenotype 4 . Based on these findings, we analysed mortality risks among phenotypes using Cox models (both unadjusted and adjusted for age) (fig. 2). The unadjusted model confirmed that phenotypes 1, 3 and 4 had a statistically significant two- to four-fold increase in mortality risk when compared with phenotype 2 . In the model adjusted for age, phenotype 1 also had a statistically significant increase in mortality risk compared with phenotype 4 (OR 3.18, 95\% CI 1.37-7.40; $\mathrm{p}=0.007$ ), and a trend towards increased mortality risk compared with phenotype 3 was also observed (OR 1.91, 95\% CI 0.94-3.06; $\mathrm{p}=0.07$ ). However, this trend did not reach statistical significance, probably due to the limited numbers of patients within these phenotypes. No significant difference in mortality risks were observed between phenotype 3 and phenotype 4 .

In conclusion, the longitudinal follow-up identified phenotype 2 as a phenotype with a very low risk of mortality. Additionally, subjects in phenotype 1 (younger subjects with severe airflow limitation, repeated exacerbations, low body mass index and low rates of cardiovascular comorbidities) had the greatest mortality rates and died at a younger age. Thus, these phenotypes actually meet the criteria required to define "clinical phenotypes". Differences in mortality between phenotypes might be even more pronounced over a longer term, but this will have to be confirmed by further survival analyses of the cohort.

Pierre-Régis Burgel*, Nicolas Roche ${ }^{\#}$, Jean-Louis Paillasseur", Isabelle Tillie-Leblond ${ }^{+}$, Pascal Chanez ${ }^{\S}$, Roger Escamilla ${ }^{f}$, Isabelle Court-Fortune ${ }^{* *}$, Thierry Perez ${ }^{+}$, Philippe Carré ${ }^{* \uparrow}$ and Denis Caillaud $^{\text {"थ }}$ on behalf of the INITIATIVES BPCO scientific committee
*Service de Pneumologie, Hôpital Cochin, AP-HP and Paris Descartes University, "Service de Pneumologie, Hôpital de l'Hôtel Dieu, AP-HP and Paris Descartes University, Paris, 'Clindatafirst, St Quentin en Yvelines, ${ }^{+}$Service de Pneumologie, Hôpital Calmette, Lille, ${ }^{\S}$ Dépt des Maladies Respiratoires, AP-HM, Université de la Méditerranée, Marseille, ${ }^{f}$ Clinique des voies respiratoires Hopital Larrey, Toulouse, **Service de Pneumologie, CHU Saint Etienne, Saint Etienne, ${ }^{\# \#}$ Service de Pneumologie, Hôpital Antoine Gayraud, Carcassonne, and "Service de Pneumologie, Hôpital Gabriel Montpied, CHU Clermont-Ferrand, France.

Correspondence: P-R. Burgel, Service de Pneumologie, Hôpital Cochin, Assistance Publique Hôpitaux de Paris, 27 rue du Faubourg St Jacques, 75679 Paris Cedex 14, France. E-mail: pierre-regis.burgel@cch.aphp.fr

Support Statement: This work was funded by unrestricted grants from Boehringer Ingelheim and Pfizer (both Paris, France).

Statement of Interest: A statement of interest for this study can be found at www.erj.ersjournals.com/site/misc/statements. xhtml

\section{REFERENCES}

1 Agusti A, Calverley PM, Celli B, et al. Characterisation of COPD heterogeneity in the ECLIPSE cohort. Respir Res 2010; 11: 122.

2 Han MK, Agusti A, Calverley PM, et al. Chronic obstructive pulmonary disease phenotypes: the future of COPD. Am J Respir Crit Care Med 2010; 182: 598-604.

3 Agusti A, Celli B. Avoiding confusion in COPD: from risk factors to phenotypes to measures of disease characterisation. Eur Respir J 2011; 38: 749-751.

4 Burgel PR, Paillasseur JL, Caillaud D, et al. Clinical COPD phenotypes: a novel approach using principal component and cluster analyses. Eur Respir J 2010; 36: 531-539.

\title{
A new point of care cotinine test for saliva to identify and monitor smoking habit
}

\section{To the Editors:}

We welcome the paper by CAPONNETTO et al. [1], in which they evaluated a nicotine-free inhalator as part of a smoking cessation programme. In the randomised controlled trial, they found no significant improvement with the inhalator, but further analysis of the data showed a significant increase in cessation rate among those with strong and very strong behavioural dependence as measured by questionnaire. While this was a good attempt to find inexpensive means to improve smoking cessation, we question their methodology to assess smoking dependency and abstinence.
Questionnaires such as those used for the Fagerström Dependence Score and the Glover-Nilsson Smoking Behavioural Questionnaire are subject to bias and intentional and unintentional underreporting. Also, smoke topography, the way a smoker uses a cigarette, depth of inhalation and nicotine metabolism are extremely variable. The best way of assessing smoking behaviour and nicotine intake is by biochemical analysis. This paper did utilise expired-air carbon monoxide (CO) monitoring, but this has significant drawbacks; it is not specific to tobacco intake (being influenced by atmospheric $\mathrm{CO}$ ) and it has a short half-life of $3 \mathrm{~h}$, so assessing smoking behaviour over a 6 - to 8 -h period. Nicotine metabolites, such as cotinine, are judged to be the best biochemical 
test for smoking behaviour [2]. Cotinine is specific to nicotine intake and has a half-life of $18 \mathrm{~h}$, meaning results are indicative of smoking over a number of days. However, cotinine measurements are largely confined to sophisticated laboratory tests, which are expensive and time consuming. We would like to take this opportunity to describe a new point of care saliva test, which measures cotinine and the other nicotine metabolites.

A previous colorimetric urine test [3] called SmokeScreen was modified. The same testing device was used, but the reagents were changed to improve sensitivity to detect the lower levels of cotinine found in saliva. The new, more sensitive assay based on the König reaction has been developed and evaluated in the laboratory and then using a group of healthy volunteers ( $n=124$, age range 21-67 yrs), including 61 smokers with a cigarette consumption of four or more cigarettes per day, (mean 16.6 cigarettes per day), 25\% of whom smoked hand rolled cigarettes.

Each provided a saliva sample using a manufactured collecting sponge and collecting bottle. $1 \mathrm{~mL}$ of saliva was eluted using the test's fixed-volume syringe. The sample was introduced onto freeze-dried reagents and quickly shaken. A sample positive for nicotine metabolites would be expected to turn pink within $1 \mathrm{~min}$, but up to $10 \mathrm{~min}$ was allowed for full colour development. The resultant colour was compared with a four-point colour chart (i.e. 1-4 point to represent weak to very intense colour) and the level of smoking recorded. Samples from nonsmokers remained unchanged. A positive colour change was obtained from 55 of the 61 smokers and a negative result from 62 of the 63 nonsmokers, giving a sensitivity of $90 \%$, specificity of $98 \%$ and accuracy of $94 \%(p<0.05)$. There is a significant difference in saliva test results between the smoking and nonsmoking groups (Chi-squared test, $\mathrm{p}<0.01$ ), indicating that the test is specific for screening smokers with a cigarette consumption of four or more cigarettes a day.

The new test was found to have a sensitivity and specificity comparable with the other commercial point of care saliva cotinine test available [4], but was quicker and is less expensive.
A dedicated colorimeter to quantify the result is under development. This test could be an important adjunct for identifying smokers and treating smoking-related diseases by providing instantaneous results at a fraction of the cost of laboratory cotinine analysis.

\section{Graham F. Cope*, Houdini H.T. Wü, Grace V. O'Donovan and Heather J. Milburn"}

*School of Medicine and Denistry, University of Birmingham, Birmingham, "School of Life Sciences, University of Warwick, Warwick, and 'Dept of Respiratory Medicine, Guy's and St Thomas' NHS Foundation Trust, London, UK.

Correspondence: G.F. Cope, School of Medicine and Denistry, University of Birmingham, Edgbaston, B15 2TT, UK. E-mail: grahamcope@btconnect.com

Statement of Interest: A statement of interest for G.F. Cope can be found at www.erj.ersjournals.com/site/misc/statements.xhtml

\section{REFERENCES}

1 Caponnetto P, Cibella F, Mancuso S, et al. Effect of a nicotine-free inhalator as part of a smoking-cessation programme. Eur Respir J 2011; 38: 1005-1011.

2 Ho MK, Mwenifumbo JC, Al Koudsi N, et al. Association of nicotine metabolite ratio and cyp2a6 genotype with smoking cessation treatment in African-American light smokers. Clin Pharmacol Ther 2009; 85: 635-643.

3 Cope G, Nayyar P, Holder R, et al. A simple near-patient test for nicotine and its metabolites in urine to assess smoking habit. Clin Chim Acta 1996; 256: 135-149.

4 Cooke F, Bullen C, Whittaker R, et al. Diagnostic accuracy of NicAlert cotinine test strips in saliva for verifying smoking status. Nicotine Tob Res 2008; 10: 607-612.

\section{Drug-resistant tuberculosis among foreign-born persons in Italy}

\section{To the Editors:}

Over the last few years, drug-resistant tuberculosis (TB) has emerged as an important threat to public health in industrialised countries. In Italy, the most recent data on resistance to the first-line drugs (FLDs) streptomycin (S), isoniazid (H), rifampicin (R) and ethambutol (E) were reported for the period 1998-2001 [1]. These studies determined the prevalence of resistance among new cases and previously treated cases, but no information was available on the contribution of immigration, which plays an important role on TB epidemiology in low-incidence countries [2].
In the last decade, while the notified incidence of TB in Italy was stable at approximately seven cases per 100,000 people annually, the proportion of foreign-born persons (FBPs) with TB increased from $22 \%$ in 1999 to $46 \%$ in 2008 [3]. In the same period, the proportion of African-born persons with TB decreased from $51 \%$ to $30 \%$, whereas the proportion of European cases increased from $16 \%$ to $33 \%$, most of them being born in Eastern Europe, including Former Soviet Union (FSU) countries.

Eastern European countries are among those with the highest TB rates caused by multidrug-resistant (MDR) Mycobacterium 